\title{
HIP 10725: The first solar twin/analogue field blue straggler ${ }^{\star}, \star \star$
}

\author{
Lucas Schirbel ${ }^{1}$, Jorge Meléndez ${ }^{1}$, Amanda I. Karakas ${ }^{2}$, Iván Ramírez ${ }^{3}$, Matthieu Castro ${ }^{4}$, Marcos A. Faria ${ }^{5}$, \\ Maria Lugaro $^{6}$, Martin Asplund ${ }^{2}$, Marcelo Tucci Maia ${ }^{1}$, David Yong ${ }^{2}$, Louise Howes ${ }^{2}$, and José D. do Nascimento Jr. ${ }^{4,7}$ \\ ${ }^{1}$ Universidade de São Paulo, Departamento de Astronomia do IAG/USP, Rua do Matão 1226, Cidade Universitária, \\ 05508-900 São Paulo, SP, Brazil \\ e-mail: lucas.schirbel@usp.br \\ 2 The Australian National University, Research School of Astronomy and Astrophysics, Cotter Road, Weston, ACT 2611, Australia \\ 3 University of Texas at Austin, McDonald Observatory and Department of Astronomy, TX 78712 Austin, USA \\ 4 Universidade Federal do Rio Grande do Norte, Departamento de Física Teórica e Experimental, 59072-970 Natal, RN, Brazil \\ 5 Universidade Federal de Itajubá, DFQ - Instituto de Ciências Exatas, 37 500-903 Itajubá, MG, Brazil \\ ${ }^{6}$ Konkoly Observatory, Research Centre for Astronomy and Earth Sciences, Hungarian Academy of Sciences, \\ Konkoly Thege Miklós út 15-17, 1121 Budapest, Hungary \\ ${ }^{7}$ Harvard-Smithsonian Center for Astrophysics, Cambridge, Massachusetts 02138, USA
}

Received 3 September 2015 / Accepted 2 October 2015

\begin{abstract}
Context. Blue stragglers are easy to identify in globular clusters, but are much harder to identify in the field. Here we present the serendipitous discovery of one field blue straggler, HIP 10725, that closely matches the Sun in mass and age, but with a metallicity slightly lower than solar.

Aims. We characterise the solar twin/analogue HIP 10725 to assess whether this star is a blue straggler.

Methods. We employed spectra with high resolution $\left(R \sim 10^{5}\right)$ and high signal-to-noise ratio (330) obtained with UVES at the VLT to perform a differential abundance analysis of the solar analogue HIP 10725. Radial velocities obtained by other instruments were also used to check for binarity. We also studied its chromospheric activity, age, and rotational velocity.

Results. HIP 10725 is severely depleted in beryllium $([\mathrm{Be} / \mathrm{H}] \leq-1.2$ dex $)$ for its stellar parameters and age. The abundances relative to solar of the elements with $Z \leq 30$ show a correlation with condensation temperature, and the neutron capture elements produced by the $s$-process are greatly enhanced, while the $r$-process elements seem normal. We found its projected rotational velocity $\left(v \sin i=3.3 \pm 0.1 \mathrm{~km} \mathrm{~s}^{-1}\right)$ to be significantly higher than solar and incompatible with its isochrone-derived age. Radial velocity monitoring shows that the star has a binary companion.

Conclusions. Based on the high $s$-process element enhancements and low beryllium abundance, we suggest that HIP 10725 has been polluted by mass transfer from an AGB star that probably had an initial mass of about $2 M_{\odot}$. The radial velocity variations suggest the presence of an unseen binary companion, probably the remnant of a former AGB star. Isochrones predict a solar-age star, but this disagrees with the high projected rotational velocity and high chromospheric activity. We conclude that HIP 10725 is a field blue straggler, rejuvenated by the mass-transfer process of its former AGB companion.
\end{abstract}

Key words. stars: abundances - stars: fundamental parameters - Sun: abundances - blue stragglers

\section{Introduction}

Blue stragglers are traditionally recognised as main-sequence stars, which are significantly bluer than the main-sequence turnoff of the population to which they belong (Ryan et al. 2001); this phenomenon has also been observed in redder stars (e.g. Sills et al. 2000). Although first observationally identified in clusters (Sandage 1953), they are also present in the field (e.g. Carney \& Peterson 1981). Field blue stragglers are harder to identify than their cluster counterparts because it is much more difficult to reliably establish which stars share a common origin in this case (since field stars that initially belonged to a cluster are already dispersed within the galaxy). However, there are other clues left

* Based on observations obtained at the European Southern Observatory (ESO) Very Large Telescope (VLT) at Paranal Observatory, Chile (observing programs 083.D-0871, 082.C-0446, 093.D-0807), and complemented with observations taken at the Observatório Pico dos Dias (OPD), Brazil (program OP2014A-011).

$\star \star$ Appendix A is available in electronic form at http://www. aanda.org behind by the blue straggler formation processes that can aid in identifying them, especially in the case of cooler stars.

Chromospheric activity in blue stragglers tends to be incompatible with their isochrone-derived ages; they present significant rotational velocities, thus appearing to be younger (Fuhrmann \& Bernkopf 1999). Severe Li depletion is also observed; this is a sign of old age (Monroe et al. 2013; Meléndez et al. 2014a) that also contradicts the young age scenario. When the phenomenon occurs through mass-transfer events (i.e., a mass exchange between a now "dead" star during its AGB phase, which pollutes its companion's photosphere, in the process also transferring angular momentum; McCrea 1964), enhancement of $s$-process elements is predicted for a sufficiently massive AGB donor, as well as abundance anomalies of light elements (Desidera et al. 2007). In this case, radial velocity variations caused by what is now a white dwarf companion are also expected. Although these properties may be found individually in normal stars, their presence alone is not an indication of the blue straggler status; when they all occur together, however, it is a strong sign in favor of this scenario (Rocha-Pinto et al. 2002). 
For solar analogues the argument can be further strengthened by analysing Be abundances. Large beryllium depletion is not observed in solar twins, where its abundance is relatively constant with age (Tucci Maia et al. 2015) ${ }^{1}$, unlike lithium, which is continuously being depleted as the star evolves (Baumann et al. 2010; Meléndez et al. 2010, 2014a; Monroe et al. 2013) by extramixing below the convective zone. This suggests that when $\mathrm{Be}$ depletion is present in these stars, other mechanisms must be responsible for it. The blue straggler scenario is a possible explanation. A small Be depletion would be due to the mass transferred by the AGB companion, as it would already be devoid of this element. However, a larger Be depletion could be produced either by enhanced internal mixing due to the transfer of angular momentum, or by a complete destruction of $\mathrm{Be}$ as a result of a merger.

In this work, we present evidence for the blue straggler status of the solar twin/analogue HIP 10725, which is the field blue straggler whose stellar parameters $\left(T_{\mathrm{eff}}, \log g\right.$, and metallicity) are the most similar to solar. We call HIP 10725 a solar twin/analogue because this star might be classified as a solar twin if the volatile elements $(\mathrm{C}, \mathrm{N}, \mathrm{O})$ are used to characterize its metallicity or as a solar analogue if the depleted refractory elements (such as iron) are used instead.

\section{Observations}

In our quest to identify new solar twins, we chose eight candidates to be studied at greater detail (HIP 1536, HIP 3238, HIP 10725, HIP 11514, HIP 106288, HIP 109381, HIP 114328, and HIP 117499), based on their colours and HIPPARCos parallaxes (i.e. their position on the colour-magnitude diagram). One of these stars (HIP 10725) showed an unusually low Be abundance, which sparked further analyses.

The observations for this sample are described in Meléndez et al. (2014a). To summarise them briefly, we observed with UVES at the VLT in dichroic mode, with the $346 \mathrm{~nm}$ setting (306-387 nm) in the blue arm and the $580 \mathrm{~nm}$ setting $(480-682 \mathrm{~nm})$ in the red arm. Most employed spectral lines are located in the red arm, where we achieved $R=110000(0.3$ arcsec slit) and a signal-to-noise ratio $(\mathrm{S} / \mathrm{N})$ of about 330 per pixel at $600 \mathrm{~nm}$. In the UV we used a slit of 0.6 arcsec, resulting in $R=65000$. A reference solar spectrum was obtained using the asteroid Juno with an identical setup. The echelle orders were extracted and wavelength calibrated using IRAF, and further processing was performed with IDL.

We also monitored radial velocities for this star to detect possible variations, and were able to acquire four measurements at different epochs. The first one was the value published by Jenkins et al. (2011) based on a spectrum obtained with FEROS at ESO taken in October 2008. Our UVES/VLT data described above were obtained in August 2009. Furthermore, we obtained two more recent measurements, one taken in July 2014 with UVES/VLT, and another one in August 2014 using the Coudé spectrograph at the Observatório Pico dos Dias (OPD) in Brazil. The radial velocities are reported in Table 1 and are discussed in Sect. 4.

Figure 1 shows part of the reduced spectra of both the Sun and HIP 10725 around the beryllium feature (top panel) and around $5320 \AA$ (bottom panel). Given the similarity in stellar

\footnotetext{
1 Only 4 stars in the sample of 118 solar analogues studied by Takeda et al. (2011) are extremely depleted in Be. The origin of this depletion is under investigation (Viallet \& Baraffe 2012; Desidera et al. 2015).
}

Table 1. Radial velocity measurements.

\begin{tabular}{lrrr}
\hline \hline $\begin{array}{l}\mathrm{MJD}^{a} \\
(\text { days })\end{array}$ & $\begin{array}{r}\text { Radial velocity } \\
\left(\mathrm{km} \mathrm{s}^{-1}\right)\end{array}$ & $\begin{array}{r}\text { Error } \\
\left(\mathrm{km} \mathrm{s}^{-1}\right)\end{array}$ & $\begin{array}{r}\text { Date } \\
(\text { yyyy-mm-dd })\end{array}$ \\
\hline 54750.270436 & -00.70 & 1.7 & $2008-10-11$ \\
55074.321678 & 69.10 & 1.0 & $2009-08-31$ \\
56844.436451 & 12.10 & 0.2 & $2014-07-06$ \\
56880.270833 & -14.50 & 1.0 & $2014-08-11$ \\
\hline
\end{tabular}

Notes. ${ }^{(a)}$ Modified Julian date.
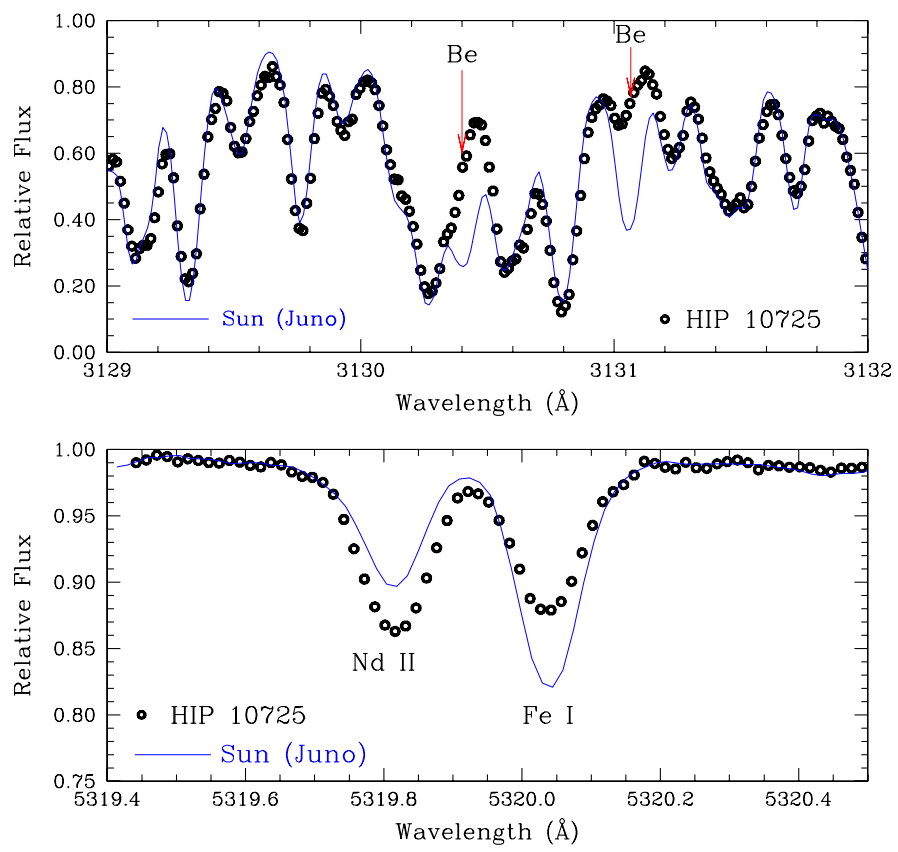

Fig. 1. Comparison of the spectra of HIP 10725 (circles) and the Sun (solid line). In the top panel, we focus on two BeII features in the UV region around $3130 \AA$. HIP 10725 shows extreme Be depletion compared to the Sun, which is not expected for solar analogues (Tucci Maia et al. 2015). The bottom panel shows an enhancement of the neutron-capture element Nd in HIP 10725 as well as a substantially lower iron content relative to the Sun.

parameters, the Be depletion in HIP 10725 is clearly visible, as well as the enhanced $\mathrm{Nd}$ abundance and lower iron content.

\section{Abundance analysis}

The same differential approach as in our previous papers (e.g. Meléndez et al. 2014a; Ramírez et al. 2014) was employed to obtain stellar parameters and chemical abundances, that is, we followed a strictly differential line-by-line analysis, using ATLAS9 model atmospheres (Castelli \& Kurucz 2004) and the 2002 version of the chemical abundance analysis program MOOG (Sneden 1973). The equivalent width (EW) measurements were performed manually using IRAF's splot task. The line list used is presented in Meléndez et al. (2014b).

The differential spectroscopic equilibrium of HIP 10725 relative to the Sun results in stellar parameters of $T_{\text {eff }}=5777 \pm 16 \mathrm{~K}$ $\left(\Delta T_{\mathrm{eff}}=+5 \pm 16 \mathrm{~K}\right), \log g=4.45 \pm 0.05 \mathrm{dex}(\Delta \log g=$ $+0.01 \pm 0.05 \mathrm{dex}),[\mathrm{Fe} / \mathrm{H}]=-0.17 \pm 0.01 \mathrm{dex}$, and a microturbulent velocity of $0.97 \mathrm{~km} \mathrm{~s}^{-1}\left(+0.07 \pm 0.04 \mathrm{~km} \mathrm{~s}^{-1}\right.$ higher than solar). The uncertainties in the stellar parameters are based on the observational uncertainties. As the stellar parameters are 
interdependent, we also took into account in the error budget this degeneracy.

With these stellar parameters, we computed differential abundances using the measured EWs, except for $\mathrm{Li}$ and $\mathrm{Be}$, which were analysed by spectral synthesis following the procedure described in Monroe et al. (2013). For Be the 2014 version of MOOG and the line list of Tucci Maia et al. (2015) were employed. Hyperfine structure was taken into account for $\mathrm{V}, \mathrm{Mn}$, $\mathrm{Co}, \mathrm{Y}, \mathrm{Ba}, \mathrm{La}, \mathrm{Pr}, \mathrm{Eu}$, and $\mathrm{Yb}$. The differential abundances are provided in Table A.1 along with the uncertainties stemming from observations (standard error in abundances) and stellar parameters. The total error was obtained by adding the observational and parameter uncertainties in quadrature.

The abundance pattern given in Table A.1 show that the average of the abundant volatile elements $\mathrm{C}, \mathrm{N}$, and $\mathrm{O}$ are within 0.1 dex of that of the Sun, but that the iron abundance is lower. This has implications for the classification of a star as either a solar twin or a solar analogue. Solar twins are classified as stars having $T_{\text {eff }}$ within $100 \mathrm{~K}$ of the solar effective temperature, and with $\log g$ and $[\mathrm{Fe} / \mathrm{H}]$ within 0.1 dex of the Sun's (Ramírez et al. 2009). $[\mathrm{Fe} / \mathrm{H}]$ is used as a proxy of metallicity because it is easier to measure than other more abundant elements such as oxygen and carbon. The definition of a solar twin can depend on the element chosen to characterise metallicity. If we consider the average of the abundant volatile elements (C, N, O), then HIP 10725 would be classified as a solar twin because its metallicity would be within 0.1 dex of that of the Sun, but if the metallicity is based on the depleted refractories (e.g. Fe), then HIP 10725 would be a solar analogue. In any case, HIP 10725 is a star that closely resembles the Sun.

We estimated the projected rotational velocity $v_{\text {rot }} \sin i$ for this star using the same procedures and line list as in Tucci Maia et al. (2015). First, we obtained the macroturbulence velocity $V_{\text {macro }}=3.6 \mathrm{~km} \mathrm{~s}^{-1}$ using the relation given in the aforementioned work. With the macroturbulence fixed, we determined $v_{\text {rot }} \sin i=3.3 \pm 0.1 \mathrm{~km} \mathrm{~s}^{-1}$ by spectral synthesis of six lines (five Fe I lines and one Ni I line). Thus the rotational velocity of HIP 10725 is significantly higher than the solar value $\left(1.9 \mathrm{kms}^{-1}\right)$.

\section{Discussion}

The abundance pattern of HIP 10725 is peculiar in the sense that we observe an excess of $s$-process elements, no enhancement of the $r$-process elements, and also a trend with condensation temperature ( $\left.T_{\text {cond }}\right)$, as can be seen in Fig. 2. This trend is usually positive in stars closely resembling the Sun, meaning that there is an overabundance of refractory elements relative to the Sun (Meléndez et al. 2009; Ramírez et al. 2009, 2010). In this star, however, we observe the opposite. In our previous works on solar twins, we speculated that the depletion of refractory elements was associated with the formation of rocky planets, but in the context of the present work, as suggested by Desidera et al. (2007), the correlation is more likely associated with dust formation in the outskirts of the former AGB companion (van Winckel 2003; Waters et al. 1992): some of the refractory elements formed dust that was removed from the gas accreted onto HIP 10725.

Figure 2 shows that the elements that depart the most from the overall $T_{\text {cond }}$ trend are those that are known to have important contributions from the $s$-process to their solar system abundances (e.g. Ba, La, Y), whereas those with the lowest $s$-process contribution to those abundances (e.g. Eu, Dy, Gd) fall very close to the $T_{\text {cond }}$ trend (see for example Simmerer et al. 2004).

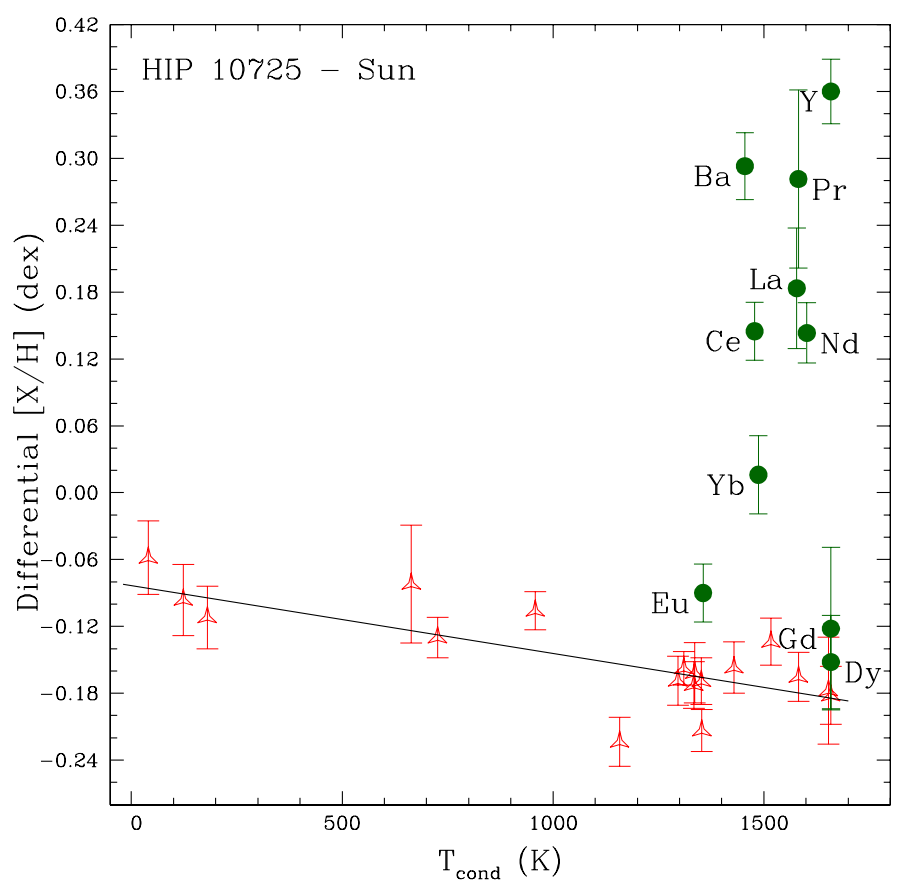

Fig. 2. Differential abundances of HIP 10725 relative to the Sun. The triangles represent the lighter elements $(Z \leq 30)$ while the filled circles represent neutron-capture elements with $Z>30$. The solid line is a fit of $[\mathrm{X} / \mathrm{H}]$ versus $T_{\text {cond }}$, taking only the lighter elements into account. The fit yields $[\mathrm{X} / \mathrm{H}]_{(\mathrm{Z} \leq 30)}=-0.0834-6.10 \times 10^{-5} T_{\text {cond }}$.

To study this peculiar abundance pattern, we followed the procedure outlined in Meléndez et al. (2014b): we fit [X/H] versus condensation temperature (Lodders 2003) for the lighter elements $(Z \leq 30)$, and subtracted this from all abundances. The fit is shown in Fig. 2 and yields $[X / H](Z \leq 30)=$ $-0.0834-6.10 \times 10^{-5} T_{\text {cond }}$, with an element-to-element scatter of 0.03 dex. Figure 3 shows the new abundance ratios after subtraction. While the $s$-process elements such as $\mathrm{Ba}, \mathrm{Nd}$, and $\mathrm{Y}$ are enhanced, the $r$-process elements Eu, Gd, and Dy are not, suggesting that indeed the pollution is due to a former AGB companion.

We modelled the pollution by the former AGB star using solar-metallicity models by Karakas (2014). We calculated the $s$-process nucleosynthesis using the same method as was outlined in Karakas (2010) and Lugaro et al. (2012). We mixed the AGB ejecta into the solar analogue's shallow surface convection zone, which we assumed to be $0.023 M_{\odot}(2.3 \%$ of the stellar mass), similar to the solar convection zone. An AGB stellar model with initial mass of $2 M_{\odot}$ provides a reasonable fit to the observed abundance pattern, as Fig. 3 shows. The amount of accreted AGB material is $0.2 \%$ of the mass lost from the $2 M_{\odot}$ AGB star over its lifetime, with most of the material lost at the tip of the AGB during the last few thermal pulses. This choice provides a fair fit to $\mathrm{Ba}, \mathrm{Nd}, \mathrm{Eu}, \mathrm{Gd}, \mathrm{Yb}$, and Dy. The predicted $\mathrm{Y}$ abundance is lower than observed, but this discrepancy might be related to the formation of ${ }^{13} \mathrm{C}$ pockets in $\mathrm{AGB}$ stars or to the activation of the ${ }^{22} \mathrm{Ne}$ neutron source in the thermal pulses (see review by Karakas \& Lattanzio 2014).

As shown above, mass transfer from an AGB star can account for the enhanced $s$-process pattern observed, as expected for blue stragglers formed by this process (Desidera et al. 2007). The donor star would subsequently have evolved into an unseen white dwarf companion. Such a companion star should be detectable through radial velocity monitoring, which is indeed the 


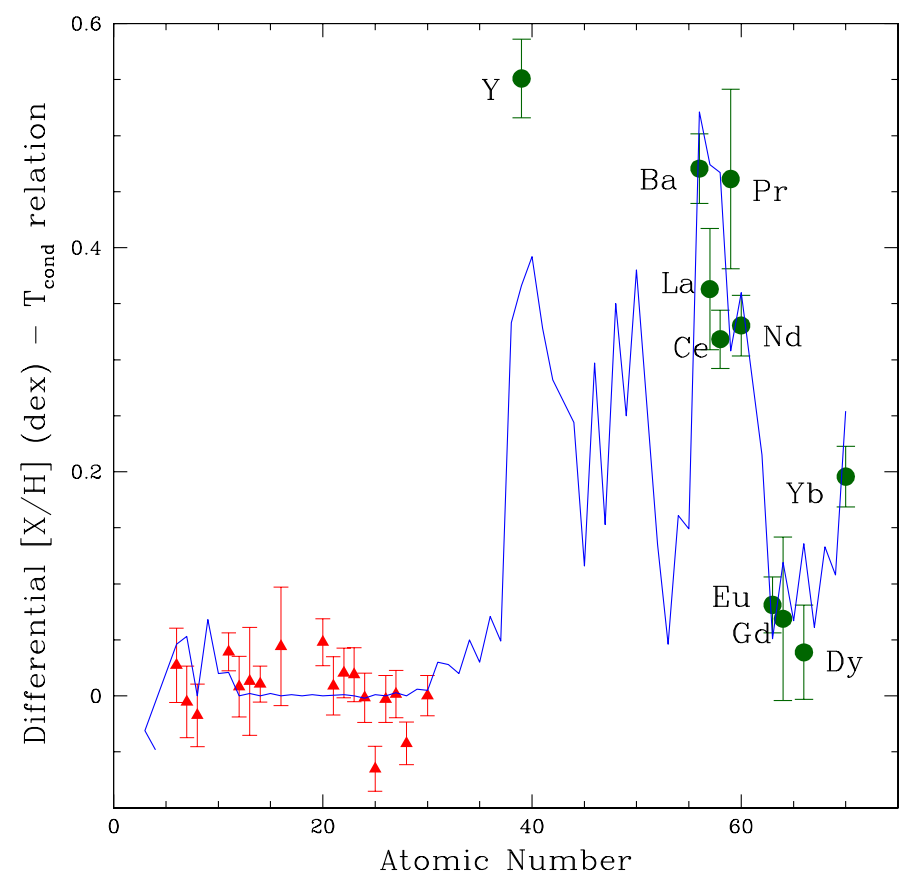

Fig. 3. Differential abundances of HIP 10725 relative to the Sun after subtracting the trend with condensation temperature. The heavier neutron-capture elements (filled circles) exhibit abundance enhancements, which are fairly well reproduced by mass transfer from an AGB star with initial mass of $2 M_{\odot}$ (solid line), except for yttrium, which is underproduced by the models.

case. As Fig. 4 shows, we found radial velocity variations of up to $\sim 70 \mathrm{~km} \mathrm{~s}^{-1}$, good evidence of the binarity of the system, which is common in field blue stragglers (Preston \& Sneden 2000; Carney et al. 2005). The radial velocities and observation dates are presented in Table 1.

In addition, we also determined $\mathrm{Be}$ abundances for HIP 10725 using spectral synthesis and found a value of $A(\mathrm{Be}) \leq 0.2 \mathrm{dex}$, or $[\mathrm{Be} / \mathrm{H}] \leq-1.2 \mathrm{dex}$, for $A(\mathrm{Be})_{\odot}=1.38 \mathrm{dex}$ (Asplund et al. 2009), meaning that the beryllium abundance in this star is at least 15 times lower than in the Sun. This is also compatible with the AGB mass transfer scenario. In the AGB scenario we propose, Be depletion would be small (0.05 dex) because of the Be-depleted material transferred by the AGB star, and large (1.15 dex) because of the transfer of angular momentum from the AGB star, which would cause the solar twin to spin up, as suggested by its higher rotational velocity.

To study the depletion of Be we used the Toulouse-Geneva Evolution Code (TGEC, Hui-Bon-Hoa 2008) including standard physics and also extra mixing beyond that predicted by standard models. We took diffusion (including gravitational settling) and rotation-induced mixing into account, as described in our previous works (do Nascimento et al. 2009; Castro et al. 2011; Tucci Maia et al. 2015). We modelled the Be depletion using the diffusion coefficient at the base of the convective zone $D_{b c z}$, and the half-height width of the tachocline $\Delta$ as parameters, which are both free parameters of stellar evolution models.

The mass transfer from the AGB star onto the surface of HIP 10725 should have enhanced the angular momentum of the convective envelope, increasing the stellar rotation. Convection transports this increase until the base of the convective zone, where a transition zone exists between the differential rotation of the convective zone and the interior, which rotates as a solid body. This transition zone, called tachocline (Spiegel \& Zahn 1992; Richard et al. 1996; Brun et al. 1998), induces a mixing

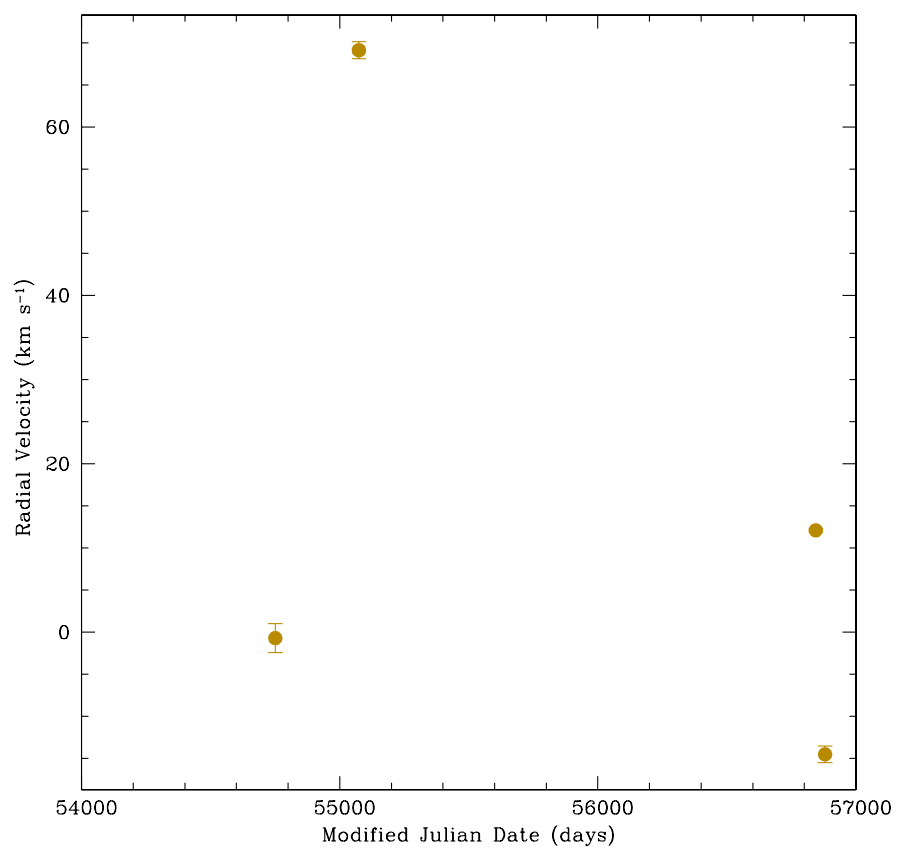

Fig. 4. Radial velocity data obtained for HIP 10725 over the course of several years, evidence for the presence of the unseen white dwarf companion.

at the base of the convective zone that can be modelled by an exponential effective diffusion coefficient $D_{b c z}$. To reproduce the observed Be depletion, we multiplied $D_{b c z}$ by 1.7 and increased the size of the tachocline $\Delta$ by a factor of 2.3. In this way, we matched the observed destruction of Be. The above changes in $D_{b c z}$ and $\Delta$, result in a surface velocity of $3.7 \mathrm{~km} \mathrm{~s}^{-1}$, which is similar to the observed $v_{\text {rot }} \sin i=3.3 \mathrm{~km} \mathrm{~s}^{-1}$.

The enhanced chromospheric activity $\left(R_{\mathrm{HK}}=-4.51\right.$, Jenkins et al. 2011) and high rotational velocity (both suggesting a young star) are incompatible with the isochrone-derived age obtained for HIP 10725 (Kim et al. 2002; Demarque et al. 2004), using probability distribution functions as in Meléndez et al. (2012). Our determined value of $v_{\text {rot }} \sin i=3.3 \mathrm{~km} \mathrm{~s}^{-1}$ suggests an age of roughly $1 \mathrm{Gyr}$, while the Yonsei-Yale isochrones result in an age of $5.2_{-2.1}^{+1.9}$ Gyr. Our upper limit for the Li abundance $(A(\mathrm{Li}) \leq$ 0.9 dex) is also about 20 times smaller than the $\mathrm{Li}$ abundance expected for an age of 1 Gyr (Tucci Maia et al. 2015), showing that the star is not young. Thus, the high projected rotational velocity is most likely the consequence of angular momentum transfer during the accretion stage from the former AGB star (Fuhrmann \& Bernkopf 1999), which "rejuvenated" HIP 10725 , enhancing both rotation and chromospheric activity.

\section{Conclusions}

We presented an observational study demonstrating that the solar analogue HIP 10725 has been polluted by $s$-process material. Its high $s$-process element abundance agrees well with mass transfer from a $2 M_{\odot}$ AGB star. A white dwarf should remain from the now defunct AGB star, and we were able to detect the presence of a companion through radial velocity variations. While the isochrone-derived age shows that its age is similar to solar, its chromospheric activity and a higher-than-usual rotational velocity would instead suggest that the star is young. This "rejuvenation" process is a consequence of mass and angular momentum transfer through wind accretion or Roche-lobe overflow during the accretion period. A large beryllium depletion compared with 
solar-type stars of similar mass and age is present as well, providing further evidence that transfer of angular momentum has taken place.

Thus, we identify HIP 10725 as a field blue straggler. Future acquisition of more radial velocity measurements will help better constrain orbital parameters and determine the mass of the probable white dwarf companion.

Finally, our work may be relevant for explaining the large depletion in beryllium observed in four of the 118 solar analogues analysed by Takeda et al. (2011), as some of these stars might also be field blue stragglers. In a recent work, Desidera et al. (2015) showed that these four Be-depleted solar analogues have binary companions, and for two of them the companion is a white dwarf, showing thus a connection with the blue straggler phenomenon.

Acknowledgements. J.M. would like to acknowledge support from FAPESP (2012/24392-2) and CNPq (Bolsa de Produtividade). L.S. acknowledges support from FAPESP (2013/25008-4) and CNPq. M.A and D.Y. acknowledge support from the Australian Research Council (grants FL110100012 and DP120100991). M.L. is a Momentum project leader of the Hungarian Academy of Sciences. M.C. acknowledges the support grant 311706/2014-2 from CNPq. A.K. was supported through an Australian Research Council Future Fellowship (FT110100475).

\section{References}

Asplund, M., Grevesse, N., Sauval, A. J., \& Scott, P. 2009, ARA\&A, 47, 481

Baumann, P., Ramírez, I., Meléndez, J., Asplund, M., \& Lind, K. 2010, A\&A, 519, A87

Brun, A. S., Turck-Chièze, S., \& Zahn, J. P. 1998, in Structure and Dynamics of the Interior of the Sun and Sun-like Stars, ESA SP, 418, 439

Carney, B. W., \& Peterson, R. C. 1981, ApJ, 251, 190

Carney, B. W., Latham, D. W., \& Laird, J. B. 2005, AJ, 129, 466

Castelli, F., \& Kurucz, R. L. 2004, Proc. IAU Symp. 210, poster A20 [arXiv: astro-ph/0405087]

Castro, M., Do Nascimento, J. D., Jr., Biazzo, K., Meléndez, J., \& de Medeiros, J. R. 2011, A\&A, 526, A17
Demarque, P., Woo, J.-H., Kim, Y.-C., \& Yi, S. K. 2004, ApJS, 155, 667

Desidera, S., Gratton, R. G., Lucatello, S., Endl, M., \& Udry, S. 2007, A\&A, 462,1039

Desidera, S., D’Orazi, V., \& Lugaro, M. 2015, A\&A, in press, DOI: $10.1051 / 0004-6361 / 201527410$

do Nascimento, J. D., Jr., Castro, M., Meléndez, J., et al. 2009, A\&A, 501, 687

Fuhrmann, K., \& Bernkopf, J. 1999, A\&A, 347, 897

Hui-Bon-Hoa, A. 2008, Ap\&SS, 316, 55

Jenkins, J. S., Murgas, F., Rojo, P., et al. 2011, A\&A, 531, A8

Karakas, A. I. 2010, MNRAS, 403, 1413

Karakas, A. I. 2014, MNRAS, 445, 347

Karakas, A. I., \& Lattanzio, J. C. 2014, PASA, 31, e030

Kim, Y.-C., Demarque, P., Yi, S. K., \& Alexander, D. R. 2002, ApJS, 143, 499 Lodders, K. 2003, ApJ, 591, 1220

Lugaro, M., Karakas, A. I., Stancliffe, R. J., \& Rijs, C. 2012, ApJ, 747, 2

McCrea, W. H. 1964, MNRAS, 128, 147

Meléndez, J., Asplund, M., Gustafsson, B., \& Yong, D. 2009, ApJ, 704, L66

Meléndez, J., Ramìrez, I., Casagrande, L., et al. 2010, Ap\&SS, 328, 193

Meléndez, J., Bergemann, M., Cohen, J. G., et al. 2012, A\&A, 543, A29

Meléndez, J., Schirbel, L., Monroe, T. R., et al. 2014a, A\&A, 567, L3

Meléndez, J., Ramírez, I., Karakas, A. I., et al. 2014b, ApJ, 791, 14

Monroe, T. R., Meléndez, J., Ramírez, I., et al. 2013, ApJ, 774, L32

Preston, G. W., \& Sneden, C. 2000, AJ, 120, 1014

Ramírez, I., Meléndez, J., \& Asplund, M. 2009, A\&A, 508, L17

Ramírez, I., Asplund, M., Baumann, P., Meléndez, J., \& Bensby, T. 2010, A\&A, 521, A33

Ramírez, I., Meléndez, J., Bean, J., et al. 2014, A\&A, 572, A48

Richard, O., Vauclair, S., Charbonnel, C., \& Dziembowski, W. A. 1996, A\&A, 312, 1000

Rocha-Pinto, H. J., Castilho, B. V., \& Maciel, W. J. 2002, A\&A, 384, 912

Ryan, S. G., Beers, T. C., Kajino, T., \& Rosolankova, K. 2001, ApJ, 547, 231

Sandage, A. R. 1953, AJ, 58, 61

Sills, A., Bailyn, C. D., Edmonds, P. D., \& Gilliland, R. L. 2000, ApJ, 535, 298

Simmerer, J., Sneden, C., Cowan, J. J., et al. 2004, ApJ, 617, 1091

Sneden, C. A. 1973, Ph.D. Thesis, 35, 28

Spiegel, E. A., \& Zahn, J.-P. 1992, A\&A, 265, 106

Takeda, Y., Tajitsu, A., Honda, S., et al. 2011, PASJ, 63, 697

Tucci Maia, M., Meléndez, J., Castro, M., et al. 2015, A\&A, 576, L10

van Winckel, H. 2003, ARA\&A, 41, 391

Viallet, M., \& Baraffe, I. 2012, A\&A, 546, A113

Waters, L. B. F. M., Trams, N. R., \& Waelkens, C. 1992, A\&A, 262, L37 


\section{Appendix A: additional table}

Table A.1. Differential abundances* of HIP 10725 relative to the Sun and errors in abundances $\Delta A$ due to observational and systematic (stellar parameters) uncertainties.

\begin{tabular}{|c|c|c|c|c|c|c|c|c|}
\hline & {$[\mathrm{X} / \mathrm{H}]$} & $\Delta A / \Delta T_{\text {eff }}$ & $\Delta A / \Delta \log g$ & $\Delta A / \Delta v_{t}$ & $\Delta A / \Delta[\mathrm{Fe} / \mathrm{H}]$ & Error & Error & Error \\
\hline Element & $\begin{array}{l}\text { LTE } \\
(\mathrm{dex}) \\
\end{array}$ & $\begin{array}{r}\Delta T_{\text {eff }} \\
+16 \mathrm{~K} \\
(\mathrm{dex}) \\
\end{array}$ & $\begin{array}{r}\Delta \log g \\
+0.05 \operatorname{dex} \\
(\operatorname{dex}) \\
\end{array}$ & $\begin{array}{r}\Delta v_{t} \\
+0.04 \mathrm{~km} \mathrm{~s}^{-1} \\
(\mathrm{dex})\end{array}$ & $\begin{array}{r}\Delta[\mathrm{Fe} / \mathrm{H}] \\
+0.01 \mathrm{dex} \\
(\mathrm{dex}) \\
\end{array}$ & $\begin{array}{r}\text { param }^{a} \\
(\mathrm{dex}) \\
\end{array}$ & $\begin{array}{l}\text { obs }^{b} \\
(\mathrm{dex}) \\
\end{array}$ & $\begin{array}{l}\text { total }^{c} \\
(\mathrm{dex}) \\
\end{array}$ \\
\hline $\mathrm{C}$ & -0.058 & -0.008 & 0.011 & 0.000 & 0.001 & 0.013 & 0.030 & 0.033 \\
\hline $\mathrm{N}$ & -0.096 & 0.022 & 0.007 & 0.008 & -0.007 & 0.025 & 0.020 & 0.032 \\
\hline $\mathrm{O}$ & -0.112 & 0.021 & 0.006 & 0.001 & -0.007 & 0.023 & 0.017 & 0.028 \\
\hline $\mathrm{Na}$ & -0.106 & 0.009 & 0.000 & -0.001 & 0.000 & 0.009 & 0.015 & 0.017 \\
\hline $\mathrm{Mg}$ & -0.162 & 0.008 & -0.004 & -0.002 & -0.001 & 0.009 & 0.026 & 0.027 \\
\hline $\mathrm{Al}$ & -0.178 & 0.008 & 0.001 & -0.001 & 0.000 & 0.008 & 0.048 & 0.048 \\
\hline $\mathrm{Si}$ & -0.158 & 0.004 & 0.004 & -0.001 & -0.001 & 0.005 & 0.015 & 0.015 \\
\hline $\mathrm{S}$ & -0.082 & -0.007 & 0.009 & -0.001 & 0.000 & 0.011 & 0.052 & 0.053 \\
\hline $\mathrm{Ca}$ & -0.134 & 0.011 & -0.007 & -0.007 & -0.001 & 0.015 & 0.015 & 0.021 \\
\hline $\mathrm{Sc}$ & -0.182 & 0.002 & 0.018 & -0.005 & -0.003 & 0.019 & 0.018 & 0.026 \\
\hline $\mathrm{Ti}$ & -0.165 & 0.016 & 0.003 & -0.004 & 0.000 & 0.017 & 0.015 & 0.022 \\
\hline V & -0.157 & 0.017 & 0.004 & -0.002 & -0.001 & 0.017 & 0.015 & 0.023 \\
\hline $\mathrm{Cr}$ & -0.169 & 0.014 & -0.001 & -0.008 & -0.001 & 0.016 & 0.015 & 0.022 \\
\hline $\mathrm{Mn}$ & -0.223 & 0.013 & -0.001 & -0.003 & 0.001 & 0.013 & 0.015 & 0.020 \\
\hline $\mathrm{Fe}$ & -0.173 & 0.013 & -0.001 & -0.007 & -0.001 & 0.014 & 0.015 & 0.021 \\
\hline Co & -0.169 & 0.012 & 0.008 & 0.002 & 0.000 & 0.015 & 0.016 & 0.022 \\
\hline $\mathrm{Ni}$ & -0.213 & 0.010 & 0.002 & -0.005 & -0.001 & 0.011 & 0.015 & 0.019 \\
\hline $\mathrm{Zn}$ & -0.130 & 0.002 & 0.005 & -0.008 & -0.002 & 0.009 & 0.015 & 0.018 \\
\hline Y & 0.360 & 0.003 & 0.008 & -0.021 & -0.022 & 0.031 & 0.015 & 0.035 \\
\hline $\mathrm{Ba}$ & 0.293 & 0.007 & 0.000 & -0.013 & -0.007 & 0.016 & 0.026 & 0.031 \\
\hline $\mathrm{La}$ & 0.183 & 0.005 & 0.029 & 0.001 & -0.005 & 0.030 & 0.045 & 0.054 \\
\hline $\mathrm{Ce}$ & 0.145 & 0.004 & 0.021 & -0.002 & -0.003 & 0.022 & 0.015 & 0.026 \\
\hline $\operatorname{Pr}$ & 0.281 & 0.031 & 0.045 & 0.025 & 0.025 & 0.066 & 0.046 & 0.080 \\
\hline $\mathrm{Nd}$ & 0.143 & 0.005 & 0.022 & -0.003 & -0.003 & 0.023 & 0.015 & 0.027 \\
\hline $\mathrm{Eu}$ & -0.090 & 0.000 & 0.018 & -0.002 & -0.002 & 0.018 & 0.017 & 0.025 \\
\hline $\mathrm{Gd}$ & -0.122 & 0.006 & 0.024 & -0.001 & -0.003 & 0.025 & 0.069 & 0.073 \\
\hline Dy & -0.152 & 0.006 & 0.022 & -0.007 & -0.004 & 0.024 & 0.035 & 0.042 \\
\hline $\mathrm{Yb}$ & 0.016 & 0.003 & 0.021 & -0.007 & -0.005 & 0.023 & 0.015 & 0.027 \\
\hline
\end{tabular}

Notes. Abundances of V, Mn, Co, Y, Ba, La, Pr, Eu, and Yb account for HFS. ${ }^{(*)} A(\mathrm{Li}) \leq 0.9$ dex, $A(\mathrm{Be}) \leq 0.2$ dex; ${ }^{(a)}$ Adding errors in stellar parameters; ${ }^{(b)}$ observational errors; ${ }^{(c)}$ total error (stellar and observational parameters). 